\title{
DE EUCALIPTO
}

\author{
Rubén A. Ananias (*) \\ Onofre Rivas (**) \\ Mario Novoa (**) \\ Magaly Garay (****)

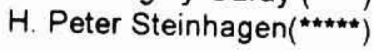

\section{RESUMEN}

secado bajo vacio con calentamiento en base de vapor sobrecalentado es una técnica que se intenta introducir en la industria maderera nacional. El presecado de madera es un método bien conocido en el pais.

En este trabajo se presentan los resultados de una experiencia exploratoria a escala industrial de secado bajo vacio, con calentamiento en base de vapor sobrecalentado, y los resultados de ensayos de presecado también a escala industrial, ambos sobre madera aserrada de $30 \mathrm{~mm}$ de espesor en eucalipto Eucalyptus globulus $L$.

La madera es recogida a la salida de la mesa de clasificación en un aserradero de la ciudad de Lota, VIII Región, Chile. El ensayo de secado bajo vacio es ejecutado en una planta DWT Moldrup de $40 \mathrm{~m} 3$ de capacidad y sistema de control automático computarizado, localizada en la ciudad de Los Angeles, VIII Región, Chile. Los 3 ensayos de presecado son desarrollados en la misma planta en que se produce la madera aserrada en un secador convencional Hildebrand HD76 de $10 \mathrm{m3}$ de capacidad y sistema de control manual. Al final de cada uno de los ensayos son medidos: la contracción, el colapso, las tensiones y los defectos del secado.

Los resultados preliminares indican que bajo vacio la madera puede secar un $25 \%$ más rápido que con una combinación de presecado y secado final hasta $10 \%$ de contenido de humedad, pero con aproximadamente el doble de contracción total tangencial y una contracción total radial mayor en un $60 \%$. La calidad del secado bajo vacio es satisfactoria. El presecado con programa variable resulta adecuado para secar eucalipto radial de $30 \mathrm{~mm}$ de espesor y largo comercial.

Palabras clave: Secado bajo vacio, presecado, eucalipto.

\footnotetext{
(*) Profesor Asistente, Depto. Ing. Maderas, Facultad Ingenierla, Universidad del Blo-Blo.

(*.*) Ingeniero Ejecución Maderas. Industrias Colcura S.A.

(*..*) Ingeniero Civil Industrias Forestales.

(.....) Profesor Asociado. U. Idaho.
} 


\section{ABSTRACT}

Vacuum drying with superheated steam is a wood drying method recently introduced in the chilean wood industry. Predrying in simplified drying chambers has been well known for many years.

This paper presents the results of an exploratory experience of vacuum drying in chilean wood industries with superheated steam and predrying, using $30 \mathrm{~mm}$ thick sawn timber of Eucalyptus globulus.

The sawnwood was collected at the outfeed of a grading conveyor line in the sawmill in Lota, VIII th Region, Chile. The vacuum drying was executed in a DWT Moldrup drier with a computarized automatic control system of $40 \mathrm{~m}^{3}$ capacity, located in Los Angeles, VIII th Region, Chile. The predrying was carried out in a conventional Hildebrand HD76 drier with manual control, of $10 \mathrm{~m}^{3}$ capacity. At the end of each of these experiments, the shrinkage, collapse, drying tension, deformations and checks were measured.

The preliminary results show that vacuum drying was $25 \%$ quicker than the combination of predrying and final drying to $10 \%$ moisture content in a kin, but resulted in nearty twice as much total shrinkage tangentially and $60 \%$ more radially. The general quality of the dried material under vacuum was acceptable. Predrying under a variable is adecuate to dry radial eucalytus of $30 \mathrm{~mm}$ thick and commercial lenght.

Keywords: Vacuum drying, predrying, Eucalyptus globulus. 


\section{INTRODUCCION}

El secado de madera bajo vacio es bien conocido desde hace varios años. Una innovación reciente, introducida por un fabricante danés, Danish Wood Treating Co.(1987), permite establecer un programa de secado con temperaturas y humedades relativas que varian durante el proceso. Esto se consigue adoptando como medio de calentamiento vapor de agua sobrecalentado. Para cada temperatura de trabajo, la presión en el autoclave se reduce debajo de la presión de vapor saturado, a un nivel que corresponde a la humedad relativa fijada por el programa de secado. El sobrecalentamiento corresponde a la diferencia entre la temperatura de trabajo y la temperatura de saturación al nivel de la presión en el autoclave.

Entre las ventajas que se evidencian en este método de secado, Simpson (1987) indica una rápida velocidad del secado y Trübswetter y Weber (1992) muestran que puede tener un bajo consumo de energía. Además, Charrier et al. (1992)

encuentran que el color natural en la superficie de maderas valiosas estéticamente puede ser mantenido. Para Chile, esto puede ser de importancia si se desea secar especies nobles tales como las provenientes del genero Nothofagus (coigue, roble, rauli o lenga), que tienen tendencia a cambiar de color por oxidación durante el proceso de secado corivencional. Igualmente Harris y Taras (1984) observan una menor variación dimensional de la madera secada bajo vacio.

Como desventajas. Trofatter et al. (1986) indican cierta heterogeneidad en la distribución de humedad en la madera. Así como también Lee y Harris (1984) detectan pequeñas reducciones en la densidad y la resistencia en compresión de la madera secada bajo vacio.

En cambio el proceso de presecado es bien conocido por la industria maderera nacional, no obstante, no es muy utilizado.

Este método se recomienda como alternativa para acelerar el secado desde verde hasta alrededor de $25 \%$ de humedad, lo que requiere una baja inversión y permite secar madera a costos atractivos para la industria. 
En este trabajo se analiza a escala industrial la calidad del secado bajo vacio en comparación al proceso de presecado en madera aserrada radial de eucalipto de $30 \mathrm{~mm}$ de espesor y largo comercial.

\section{METODOLOGIA}

\section{Secado Bajo Vacio.}

Se ejecuta un ensayo de secado bajo vacio en un secador DWT Moldrup con sistema de control automatico computarizado y de $40 \mathrm{~m} 3$ de capacidad. El secador es un autoclave de una via que tiene un diametro interno de 4 metros y un largo de 20,5 metros.

La madera aserrada es recogida al azar a la salida de la mesa de clasificación de un aserradero en Lota (Octava Región, Chile). Luego es trasladada a una planta industrial de Los Angeles (Octava Región, Chile).

Alli la madera es encastillada en el autoclave, formando una carga con 6 castillos de 4 lingas cada uno. Son usados 7 separadores de $25 \mathrm{~mm}$ de espesor por camada de madera.

Una linga compuesta por 170 piezas de $1^{\prime \prime} \times 4^{\prime \prime} \times 10^{\prime}$ es utilizada para medir los defectos al empezar y terminar el proceso, esta linga se ubica al azar entre los castillos.

Se preparan 48 muestras de $76 \mathrm{~cm}$ de largo, extraidas de 48 piezas diferentes, para medir contenido de humedad inicial y final, gradiente de humedad, variación dimensional y tensiones al final del secado. Se distribuyen aleatoriamente 8 muestras en cada castillo de madera.

Se mide solo humedad inicial y final por método gravímetrico, en base a 2 probetas obtenidas de los extremos de cada muestra; se emplea norma chilena of 84 176/1. Se determina la densidad básica según noma chilena of 86 176/2. La contracción se mide en tres puntos en el centro de los cantos y en cuatro puntos en el centro de las caras; se utiliza norma chilena of 84 176/3. El colapso se mide en base a una estimación visual adaptada de la metodologia propuesta por Kauman 
(1966). Las tensiones de secado son medidas en base de la desviación de los dientes de probetas tipo tenedor y evaluadas según metodologia propuesta por INFOR (1987). Los defectos del secado se miden segun norma chilena of 74992 y 993. La calidad del secado se estima en base a un indice ponderado de defectos propuesto por Kauman (1966).

Una falla en el sistema de retomo del condensado impide hacer uso del sistema de control automatico computarizado del secador. La duración del secado es estimado por la cantidad de agua que puede extraerse del volumen de madera en proceso y el tiempo transcumido en régimen para alcanzar la máxima cantidad de agua efectiva que registra el aparato.

\section{Presecado y Secado al Aire}

Se ejecutan tres ensayos de presecado y un ensayo de secado natural, incluyendo en todos un proceso de secado convencional final. En este caso la madera aserrada es recogida de la misma manera y en el mismo aserradero anterior (Lota, Octava Región, Chile).

Los ensayos de presecado son realizados en la misma planta, en un secador Hildebrand HD76 con sistema de control manual y de $10 \mathrm{~m}^{3}$ de capacidad, con una sola via y 7,6 m de largo. La velocidad del aire promedio medida entre las piezas de madera es de $1,5 \mathrm{~m} / \mathrm{s}$.

Son encastilladas a mano en cada ensayo 994 piezas de $1^{\prime \prime} \times 5^{\prime \prime} \times 12^{\prime}$. Son usados 7 separadores de $25 \mathrm{~mm}$ por camada de madera. Se emplean 142 piezas de 1" × 5" × 12' para evaluar los defectos del secado al iniciar y terminar cada proceso. Se preparan 21 muestras para la conducción del presecado.

Además, se prepara un castillo de secado natural durante el verano de 1989 en el patio de secado de la planta industrial (Lota, Octava Región Chile).

En cada uno de estos ensayos de presecado y de secado al aire se concluye con un secado convencional final, en un secador Moore de $60 \mathrm{~m}^{3}$ de capacidad y velocidad del aire promedio de $1,2 \mathrm{~m} / \mathrm{s}$.

Las mediciones y evaluaciones de humedad, contracción, colapso, tensiones y calidad del secado son ejecutadas de la misma manera que se indica anteriormente para el ensayo de secado bajo vacio. 
Los programas de secado se muestran en el Cuadro $N^{\circ} 1$. El programa de secado bajo vacio es obtenido de Novoa y Rivas (1990), los programas de presecado se recogen de Garay (1991) y el programa de secado convencional proviene de Teuber (1990).

En el sistema de secado bajo vacio DWT Co. (1987), para una temperatura ambiente $T$, la presión en el autoclave $p$ es una fracción de la presión de saturación que corresponde a la humedad relativa HR deseada. Se obtiene un efecto de programa de secado al cambiar la temperatura y la humedad relativa oportunamente.

El programa de secado bajo vacio incluye un tratamiento final para favorecer el alivio de tensiones de secado. El programa de presecado tiene condiciones ambientales de temperatura y humedad relativa del mismo orden que el secado natural programa de verano. Este puede ser más rápido dada la circulación forzada del aire en las camaras. El programa de presecado variable presenta la ventaja de mantener la temperatura del bulbo húmedo constante, lo que facilita su conducción. El secado convencional final, incluye tratamientos de acondicionamiento intermedio y acondicionamiento final a una temperatura de $60^{\circ} \mathrm{C}$ en ambiente saturado. 


\section{Cuadro $\mathrm{N}^{\circ} 1$}

PROGRAMAS SECADO. EUCALIPTO $30 \mathrm{MM}$.

\begin{tabular}{|c|c|c|c|c|c|}
\hline & $\begin{array}{c}\mathrm{CH} \\
(\%)\end{array}$ & $\begin{array}{c}\mathrm{T} \\
\left.{ }^{\circ} \mathrm{C}\right)\end{array}$ & $\begin{array}{c}\mathrm{HR} \\
(\%)\end{array}$ & $\begin{array}{c}\mathrm{CHE} \\
(\%)\end{array}$ & $\begin{array}{c}\text { P } \\
(\text { bar })\end{array}$ \\
\hline \multirow{4}{*}{ S.V. } & verde & 40 & 90 & 25,0 & 0,066 \\
& 50 & 42 & 84 & 22,0 & 0,068 \\
& 30 & 48 & 77 & 15,0 & 0,085 \\
& 20 & 51 & 70 & 12,0 & 0,090 \\
& 15 & 60 & 59 & 7,0 & 0,116 \\
\hline Pre 1 & 10 & 68 & 59 & 5,0 & 0,166 \\
\hline & verde-20 & 30 & 68 & 12,0 & \\
Pre 2 & 50 & 35 & 81 & 16,0 & \\
& 40 & 38 & 62 & 10,5 & \\
& 30 & 40 & 57 & 9,5 & \\
\hline S.N. & 25 & 43 & 42 & 7,0 & \\
\hline S.C. & verde-20 & 20 & 70 & 15,0 & \\
& 20 & 42 & 88 & 19,5 & \\
& a.i. & 60 & 100 & 26,5 & \\
& 10 & 62 & 46 & 6,0 & \\
& a.f. & 62 & 100 & 26,5 & \\
\hline
\end{tabular}

Donde,

$\begin{array}{lll}\text { CH } & \text { contenido humedad. } \\ \text { T } & \text { temperatura ambiente. } \\ \text { HR } & \text { humedad relativa. } \\ \text { CHE } & \text { contenido humedad equilibrio. } \\ \text { P } & \text { presión en el autoclave. } \\ \text { S.V } & \text { secado bajo vacio. } \\ \text { Pre 1 } & \text { presecado constante. } \\ \text { Pre 2 } & \text { presecado variable. } \\ \text { S.N } & \text { secado natural. } \\ \text { S.C. } & \text { secado convencional. } \\ \text { a.i. } & \text { acondicionamiento intermedio. } \\ \text { a.f. } & \text { acondicionamiento final. }\end{array}$




\section{RESULTADOS Y DISCUSION}

C.H. $(\%)$

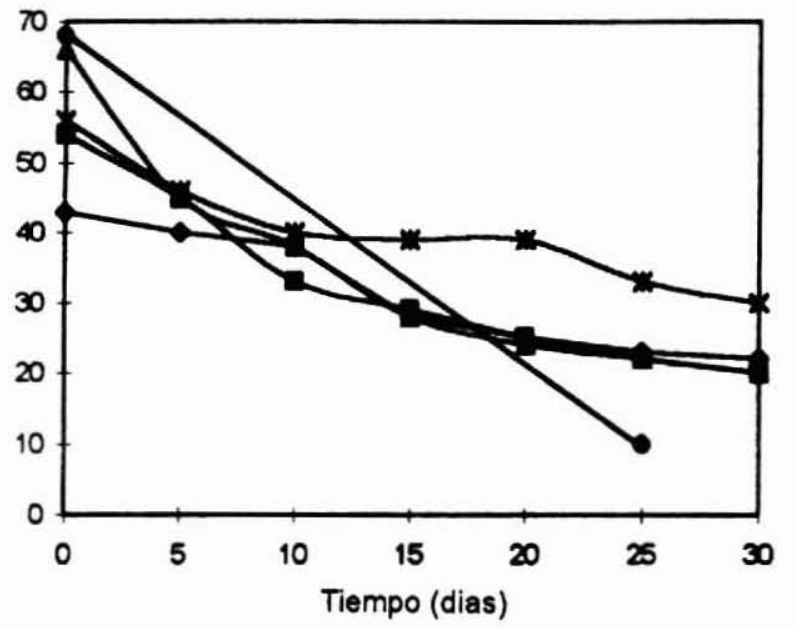

$\sim P r e 1-P r e 2-P r e 2 R-8 . V .-m-8 . N$.

Figura N 01 . CURVAS DE SECADO.

EUCALIPTO RADIAL $30 \mathrm{~mm}$

En la Figura $\mathrm{N}^{0} 1$ se observa la rápida velocidad del presecado variable que presenta el mismo orden de magnitud que el secado bajo vacio, especialmente al inicio del secado. No obstante, al considerar sólo los contenidos de humedad inicial y final en el ensayo de secado bajo vacio, las comparaciones son limitadas.

Como se menciona anteriormente, el programa de secado convencional, con el que finalizan todos los ensayos de presecado, incluye 8 horas de un acondicionamiento intermedio en ambiente saturado a $60^{\circ} \mathrm{C}$, para favorecer la recuperación del colapso, y un acondicionamiento final similar para el alivio de las tensiones de secado. No queda claramente establecido si estos tratamientos 
favorecen además la velocidad del secado, debe seguir investigandose este aspecto.

Se observa en el Cuadro $N^{\circ} 2$ una notable heterogeneidad en el contenido de humedad inicial de la madera secada bajo vacio, la cual es reducida al final. Esto pudo ser consecuencia del tratamiento de acondicionamiento final de 4 horas dado a la madera.

\section{Cuadro $\mathrm{N}^{\circ} 2$}

\section{DISTRIBUCION CONTENIDO HUMEDAD DURANTE SECADO. EUCALIPTO RADIAL 30 MM.}

\begin{tabular}{|c|c|c|}
\hline Método & $\begin{array}{c}\text { CHf (std) } \\
(\%)\end{array}$ & $\begin{array}{c}\text { CHi (std) } \\
(\%)\end{array}$ \\
\hline S.V. & $10,1(3,6)$ & $68,3(18,3)$ \\
\hline Pre 1 & $21,6(5,6)$ & $41,5(10,0)$ \\
Pre 1+S.C. & $11,3(5,6)$ & $21,6(5,6)$ \\
\hline Pre 2 & $18,0(4,5)$ & $54,2(13,8)$ \\
Pre 2+ S.C. & $10,1(1,0)$ & $18,0(4,5)$ \\
Pre 2R & $21,6(5,6)$ & $65,2(20,1)$ \\
Pre 2R + S.C. & $11,3(5,6)$ & $21,6(5,6)$ \\
\hline S.N. & $21,8(2,9)$ & $56,3(13,6)$ \\
S.N. + S.C. & $13,0(1,9)$ & $21,8(2,9)$ \\
\hline
\end{tabular}

$\mathrm{CHf}=$ Contenido humedad final, $\mathrm{CHi}=$ contenido humedad inicial, std = Desviación standar .

Cuadro $\mathrm{N}^{\circ} 3$

CONTRACCION DEL SECADO. EUCALIPTO RADIAL 30 MM

\begin{tabular}{|c|c|c|}
\hline Método & $\begin{array}{c}\text { Tangencial } \\
(\%)\end{array}$ & $\begin{array}{c}\text { Radial } \\
(\%)\end{array}$ \\
\hline S.V. & 14,5 & 8,2 \\
\hline Pre 1 & 10,0 & 4,6 \\
Pre 1+ S.C. & 11,7 & 5,3 \\
\hline Pre 2 & 7,9 & 4,0 \\
Pre 2+ S.C. & 7,8 & 5,2 \\
\hline Pre 2R & 6,9 & 3,8 \\
Pre 2R + S.C. & 7,3 & 4,9 \\
\hline S.N. & 8,1 & 3,3 \\
S.N. + S.C. & 11,8 & 5,5 \\
\hline
\end{tabular}


En el Cuadro $\mathrm{N}^{\circ} 3$ se observa que la madera secada bajo vacio muestra la mayor variación dimensional, lo cual puede ser consecuencia de limitaciones del programa de secado empleado lo que induce la presencia de colapso más pronunciada en esta madera, tal como se indica en el Cuadro $N^{\circ} 4$.

Por otra parte, las diferencias en el contenido de humedad inicial en cada uno de los procesos que se indican en el Cuadro $\mathrm{N}^{\circ} 2$, no parecen contribuir con el nivel del colapso observado en el ensayo de secado bajo vacio, ya que el contenido de humedad inicial en este proceso es de $68 \%$ y la contracción tangencial y radial son casi el doble de la obtenida en los ensayos de presecado variable. El ensayo de presecado constante, que tiene un contenido de humedad inicial de $42 \%$, presenta una mayor contracción que los ensayos de presecado variable, que tienen más alto contenido de humedad inicial. Esto puede evidenciar cierto efecto del método de secado bajo vacio sobre el nivel del colapso mostrado en el Cuadro $\mathrm{N}^{\circ} 4$.

En el Cuadro $\mathrm{N}^{0} 3$ se observa que con el programa de presecado variable se obtiene la menor variación dimensional, incluso menor que en la madera secada naturalmente. La misma tendencia se observa después del secado convencional final. La minimas diferencias, entre la contracción al final de los ensayos de presecado en comparación a la contracción después del secado convencional, son debidas a la efectividad de los trtatamientos de acondicionamiento intermedio en ambiente saturado para recuperar parte del colapso de la madera. En cambio este efecto no es notorio en la madera secada naturalmente. 


\section{Cuadro $N^{\circ} 4$}

COLAPSO DEL SECADO EN PORCENTAJE. EUCALIPTO RADIAL 30 MM.

\begin{tabular}{|c|c|c|c|c|}
\hline Metodo & s/c & Leve & Medio & Intenso \\
\hline S.V. & 50 & 59 & 45 & 16 \\
\hline Pre 1 & 27 & 40 & 17 & 16 \\
Pre 1+S.C. & 34 & 29 & 33 & 5 \\
\hline Pre 2 & 43 & 33 & 20 & 4 \\
Pre 2+ S.C. & 44 & 38 & 17 & 1 \\
\hline Pre 2R & 72 & 20 & 8 & 0 \\
Pre 2R + S.C. & 71 & 18 & 10 & 1 \\
\hline S.N. & 55 & 29 & 15 & 1 \\
S.N. + S.C. & 42 & 36 & 20 & 2 \\
\hline
\end{tabular}

$\mathrm{s} / \mathrm{c}=\mathrm{Sin}$ colapso

En el Cuadro $\mathrm{N}^{\circ} 4$ se observa que el colapso esta presente en todas las modalidades de secado del eucalipto, incluyendo el secado natural. En el ensayo en que se repite el programa variable se obtiene el colapso menos pronunciado, esto es del orden de un $70 \%$ de piezas libres de colapso. Por lo anterior, durante el secado de eucalipto debe considerarse una cierta pérdida de volumen como consecuencia de su tendencia al colapso, la cual puede minimizarse con una buena práctica de secado, una adecuada selección de la madera y una mejor selección del método de secado. 


\section{Cuadro $N^{\circ} 5$}

Tensiones de secado en porcentaje. Eucalipto radial $30 \mathrm{~mm}$.

\begin{tabular}{|c|r|r|r|c|}
\hline Método & s/t & Leve & Medio & Intenso \\
\hline S.V. & 9 & 2 & 22 & 67 \\
\hline Pre 1 & 17 & 50 & 33 & 0 \\
Pre 1+S.C & 0 & 26 & 26 & 48 \\
\hline Pre 2 & 0 & 33 & 33 & 34 \\
Pre 2+ S.C. & 7 & 7 & 53 & 33 \\
\hline Pre 2R & 20 & 40 & 0 & 40 \\
Pre 2R+S.C. & 12 & 35 & 41 & 12 \\
\hline S.N. & 0 & 60 & 40 & 0 \\
S.N. + S.C. & 12 & 29 & 35 & 24 \\
\hline
\end{tabular}

$\mathbf{s} / \mathbf{t}=$ Sin tensión de secado

En el Cuadro $\mathrm{N}^{\circ} 5$ se aprecia la intensidad de las tensiones durante el secado de eucalipto radial de $30 \mathrm{~mm}$. Se observa que esta es de magnitud comparable en todos los metodos ensayados hasta una intensidad media, no obstante la proporción de madera con tensiones de secado en el secado bajo vacio es del orden de un $70 \%$. La magnitud de las tensiones en el secado bajo vacio se puede apreciar en la Figura $\mathrm{N}^{\circ} 2$. En cambio en el presecado esto no supera el $50 \%$. Se estima que esto puede ser mejorado introduciendo variaciones en el tratamiento de acondicionamiento final durante el secado por vacio, especialmente en la duración de este tratamiento. 

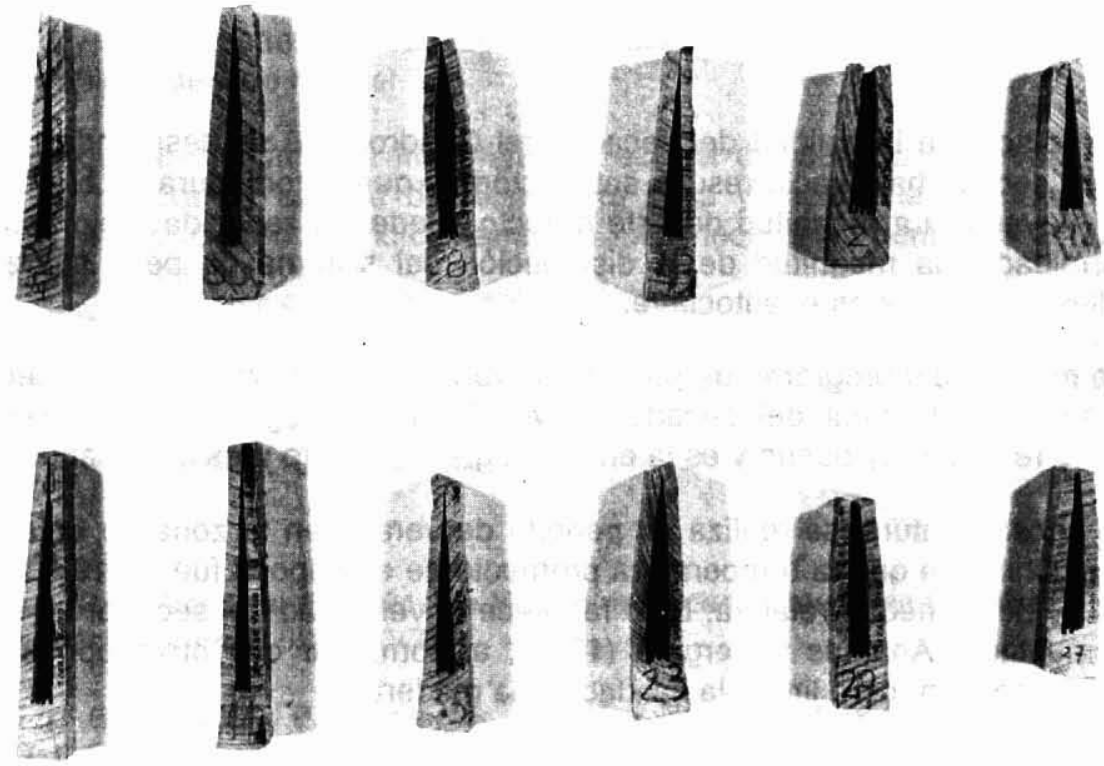

Figura No 2. FOTOGRAFIAS PROBETAS TIPO TENEDOR

FINAL SECADO BAJO VACIO

EUCALIPTO RADIAL $30 \mathrm{~mm}$

Cuadro $N^{0} 6$

CALIDAD DEL SECADO. EUCALIPTO RADIAL $30 \mathrm{~mm}$.

\begin{tabular}{|c|cccc|c|c|}
\hline Método & Arque & Enco & Torce & Aca & Grie & Calidad \\
\hline S.V. & 0,03 & 0,12 & 0,82 & 0,00 & 0,17 & 1,14 \\
\hline Pre 1 & 0,25 & 0,29 & 0,02 & 0,15 & 0,34 & 1,03 \\
Pre 1 + S.C. & 0,15 & 0,13 & 0,05 & 0,22 & 0,29 & 0,84 \\
Pre 2 & 0,09 & 0,23 & 0,04 & 0,02 & 0,08 & 0,46 \\
Pre 2+ S.C. & 0,12 & 0,23 & 0,04 & 0,02 & 0,04 & 0,45 \\
\hline Pre 2R & 0,06 & 0,29 & 0,01 & 0,04 & 0,12 & 0,52 \\
Pre 2R + S.C. & 0,04 & 0,28 & 0,01 & 0,01 & 0,07 & 0,41 \\
\hline S.N & 0,16 & 0,21 & 0,06 & 0,05 & 0,31 & 0,79 \\
S.N. + S.C. & 0,25 & 0,29 & 0,03 & 0,15 & 0,40 & 1,12 \\
\hline
\end{tabular}

donde,

arque $=$ Arqueadura, enco $=$ Enconvadura, torce $=$ Torcedura,

aca $=$ Acanaladura, grie $=$ Grietas 
En relación a la calidad del secado, del Cuadro $N^{0} 6$ se desprende que la madera secada bajo vacio resulta satisfactoria y que la torcedura es el defecto más relevante. La magnitud de este defecto puede ser reducida mejorando la uniformidad y la magnitud de la distribución del sistema de peso sobre los castillos de madera en el autoclave.

La madera del programa de presecado variable resulta mejor que la secada naturalmente. Al final del secado convencional el programa de presecado variable resulta muy bueno y es la encorvadura el defecto mas importante.

El secado natural se realiza en periodo de verano en la zona de Lota, VIII región, Chile, en que la temperatura promedio de esa época fué de $19^{\circ} \mathrm{C}$ con un $64 \%$ de humedad relativa. Esto favorece la velocidad del secado, tal como lo demuestran Ananias y Vergara (1991), al comparar con otras epocas del inicio del secado, pero limita la calidad de la madera.

\section{CONCLUSIONES}

De acuerdo a una experiencia preliminar a escala industrial, el secado bajo vacio con calentamiento a base de vapor sobrecalentado permite secar más rapidamente, pero con mayor colapso la madera aserrada en $30 \mathrm{~mm}$ de espesor de eucalipto radial, desde verde a $10 \%$ de humedad final. Se requiere de un análisis técnico-económico para averiguar si la mayor velocidad del secado justifica el mayor costo y complejidad de una instalación de secado bajo vacio.

El presecado variable, desde verde a $20 \%$ de humedad, en combinación con un secado convencional posterior hasta $10 \%$ de humedad final, permite secar adecuadamente madera aserrada de eucalipto radial de $30 \mathrm{~mm}$ de espesor. Se minimiza el tiempo de secado y se logra una muy buena calidad de la madera seca.

El secado bajo vacio presenta una mayor variación dimensional, como consecuencia de la abundancia de colapso. La pérdida de dimensiones de la 
madera y las tensiones del secado en la madera presecada son de magnitud comparable al secado natural.

Se requiere continuar la investigación acerca del secado bajo vacio para tratar de mejorar la calidad de la madera seca y reducir la abundancia de colapso, introduciendo acondicionamiento intermedio y aumentando el tiempo del acondicionamiento final.

\section{REFERENCIAS BIBLIOGRAFICAS}

Ananias, R.A.; Vergara, F., 1991. Un Modelo de Secado Natural de Eucalipto. Ciencia e Investigación Forestal 5(2):230-236

Charrier, B.; Haluk, J.P.; Janin, G., 1992. Prevention of Brown Discoloration in European Oakwood Ocurring During Kiln Drying by a Vacuum Process: Colorimetric Comparative Study with a Traditional Process. Holz Roh-Werkstoff 50 (11): 433-437.

Danish Wood Treating Co., 1987. The DWT Moldrup Drying Process. Cátalogo p. 1015.

Garay, M., 1991. Presecado Industrial de Eucalipto. Proyecto Título, Depto. Ing. Maderas, Facultad Ingeniería, U. Bío-Bio, Concepción, Chile.

Harris, R.A.; Taras, M.A., 1984. Comparison of Moisture Contents Distribution, Stress Distribution, and Shrinkage of Red Oak Lumber Dried by Radio Frecuency/Nacuum Drying Process and a Conventional Kiln. Forest Prod. J. 34 (1): 44-54.

INFOR, 1987. Secado por Deshumidificación de Especies Madereras de Interés Comercial. Informe Tecnico $N^{\circ} 100$.

Kauman, W.G., 1966. Ensayos de Secado en Coigue. Informe Técnico $N^{\circ} 25$, Instituto Forestal, Santiago, Chile.

Novoa, M.; Rivas, O., 1990. Secado por Vacio: Una Experiencia con Eucalyptus globulus. Seminario Titulación, Depto. Ing. Maderas, Facultad Ingenierla, U. Bio-Bio, Concepción, Chile.

Simpson, W.T., 1987. Vacuum Drying Northern Red Oak. Forest Prod. J. 37 (1): 35-38. 
Teuber, P., 1990. Mejoramiento del Secado Industrial de Eucalipto. Proyecto Título, Depto. Ing. Maderas, Facultad Ingeniería, U. Bío-Bio, Concepción, Chile.

Trofatter, G.; Harris, R.A.; Schroeder, J.; Taras, M.A., 1986. Comparison of Moisture Contents Variation in Red Oak Lumber Dried by Radio Frecuency/Nacuum Process and a Conventional Kiln. Forest Prod. J. 36 (5): 25-28.

Trubswetter, T.; Weber, M., 1992. Untersuchungen an Finer DWT MoldrupTrockenanlage. Holz Roh-Werkstoff 50 (7/8): 286-290. 